\title{
Palifermin as Primary Prophylaxis for Mucositis Prevention in Patients with B-Cell Non-Hodgkin Lymphoma
}

\author{
Anthony Zembillas ${ }^{1}$, Stefanie Thomas $^{2}$, seth $\operatorname{rotz}^{2}$, Ilia Buhtoiarov ${ }^{1}$, and Rabi Hanna ${ }^{1}$ \\ ${ }^{1}$ Cleveland Clinic \\ ${ }^{2}$ Cleveland Clinic Foundation
}

October 22, 2020

Palifermin as Primary Prophylaxis for Mucositis Prevention in Patients with B-Cell Non-Hodgkin Lymphoma Anthony S. Zembillas, PharmD, BCPS, BCOP ${ }^{1,3}$, Stefanie M. Thomas, MD, MS ${ }^{2}$, Seth J. Rotz MD ${ }^{2}$, Ilia N. Buhtoiarov, $\mathrm{MD}^{2}$, Rabi Hanna, $\mathrm{MD}^{2}$

(1) Pharmacy, Cleveland Clinic, Cleveland, OH;

(2) Department of Pediatric Hematology Oncology and Bone Marrow Transplantation, Cleveland Clinic Children's, Cleveland, OH;

(3) Address correspondence to:

Funding Source: Supported in part by a grant from the NIH NCATS (2KL2TR002547 PI Dweik, to S.J.R.)

To the Editor:

Progress continues to be made regarding the treatment of children and adolescents with aggressive B-cell Non-Hodgkin Lymphoma (NHL). Recent data demonstrated patients with mature B-cell NHL have an improved overall survival with the addition of rituximab to lymphomes malins B (LMB) chemotherapy. ${ }^{1}$ However, these patients experienced significant therapy-related adverse events including febrile neutropenia, stomatitis, and infection. Oral mucositis, the second most common adverse event, occurred in up to $80 \%$ of patients with grade 3 oral mucositis occurring in $71 \%$ of patients. ${ }^{1}$ Complications related to mucositis include significant morbidities such as severe pain requiring opioids, nutritional deficiencies necessitating parenteral nutrition, life-threatening bloodstream infections, and prolonged hospitalizations likely increasing the overall cost of care. ${ }^{2}$ Proper oral care is the standard first-line approach to mucositis prevention but its benefits may be limited with more intensive chemotherapy regimens.

Palifermin, a human recombinant keratinocyte growth factor, is a preventative medication that has shown positive effects on reducing mucositis frequqncy and severity in children receiving cancer therapy. ${ }^{3}$ Studies of palifermin most frequently involve patient's undergoing hematopoietic stem cell transplant (HSCT) with limited data in the non-HSCT setting. ${ }^{4-6}$ Data are encouraging regarding the use of palifermin as secondary prophylaxis, showing effectiveness in children and adolescents with NHL who previously developed mucositis. ${ }^{6}$ This data, combined with our experience of significant toxicity in our patient population led us to administer palifermin as primary prophylaxis for mucositis prevention in patients receiving Group B or Group C LMB therapy for B-cell NHL, as part of routine clinical practice.

We first utilized this approach in a young adult male with Stage II A diffuse large B cell lymphoma (DLBCL) treated per Group B of ANHL1131. He received a single dose of palifermin $180 \mathrm{mcg} / \mathrm{kg}$ IV in the outpatient setting approximately three days prior to cycles 1 and 2 . He did not receive palifermin prior to cycle 3 due to the perceived lower risk of mucositis. But after developing severe mucositis requiring opioid analgesics during 
cycle 3, palifermin was reinstituted prior to cycle 4 . Of the three cycles where palifermin was used, there were no mucositis-related complications. The patient remains in complete remission one year post-therapy. The second patient was a young adult female with Burkitt lymphoma treated per Group C1 of ANHL1131. She received a single dose of palifermin $180 \mathrm{mcg} / \mathrm{kg}$ IV in the outpatient setting approximately 3 days prior to all 6 cycles of chemotherapy. She did not have mucositis-related complications or require opioids for mucositis-related pain throughout her entire treatment. She has no evidence of disease at end of therapy. No toxicities from palifermin were noted in either patient.

Our experience suggests that palifermin is a potential therapeutic option as primary prophylaxis for NHL patients receiving high intensity chemotherapy commonly causing grade 3-4 mucositis. Prospective studies are warranted to confirm the benefit and safety of palifermin compared to other interventions such as oral saline rinses or a standardized oral mouth care protocol.

\section{References}

1. Minard-Colin V, Auperin A, Pillon M, Burke GAA, Barkauskas DA, Wheatley K. Rituximab for highrisk, mature B-cell non-Hodgkin's lymphoma in children. N Engl J Med 2020;382:2207-2219.

2. Spielberger R, Stiff P, Bensinger W, Gentile T, Weisdorf D, Kewalramani T. Palifermin for Oral Mucositis after Intensive Therapy for Hematologic Cancers. N Engl J Med2004;351:2590-2598.

3. Mazhari, F, Shirazi AS, Shabzendehdar M. Management of oral mucositis in pediatric patients receiving cancer therapy: a systematic review and meta-analysis. Pediatr Blood Cancer 2019;66:e27403.

4. Vadhan-Raj S, Trent J, Patel S, Zhou X, Johnson MM, Araujo D. Single-dose palifermin prevents severe oral mucositis during multicycle chemotherapy in patients with cancer. Ann Intern Med 2010;153:358367 .

5. Saber W, Zhang MJ, Steinert P, Chen Min, Horowitz MM. The impact of palifermin use on hematopoietic cell transplant outcomes in children. Biol Blood Marrow Transplant 2016;22:1460-1466.

6. Dazhi L, Seyboth B, Mathew S, Gilheeney SW, Chou AJ, Drill E. Retrospective evaluation of palifermin use in nonhematopoietic stem cell transplant pediatric patients. J Pediatr Hematol Oncol 2017;39:177182. 